CRYSTALLOGRAPHIC COMMUNICATIONS

ISSN 2056-9890

Received 4 December 2014

Accepted 7 December 2014

Edited by H. Stoeckli-Evans, University of Neuchâtel, Switzerland

Keywords: crystal structure; bisphosphonate; polymorphism; minodronic acid; hydrogen bonds; redetermination

CCDC reference: 1038087

Supporting information: this article has

supporting information at journals.iucr.org/e

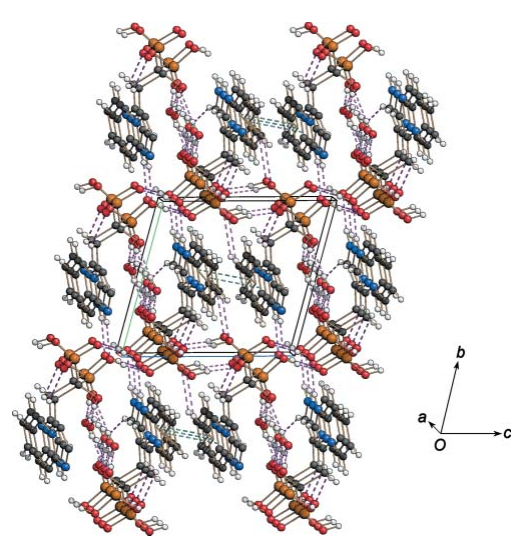

OPEN $\odot$ ACCESS

\section{Crystal structure of zwitterionic 3-(2-hydroxy-2- phosphonato-2-phosphonoethyl)imidazo[1,2-a]- pyridin-1-ium monohydrate (minodronic acid monohydrate): a redetermination}

\author{
Annalisa Airoldi, ${ }^{a}$ Piergiorgio Bettoni, ${ }^{a}$ Monica Donnola, ${ }^{a}$ Gianluca Calestani ${ }^{b}$ and \\ Corrado Rizzoli ${ }^{\mathbf{b} *}$ \\ ${ }^{\mathbf{a}}$ R\&D Division, PROCOS S.p.A., Via G. Matteotti 249, 28062 Cameri (Novara), Italy, and ${ }^{\mathbf{b}}$ Universitá degli Studi di \\ Parma, Dipartimento di Chimica, Parco Area delle Scienze 17/A, 43124 Parma, Italy. *Correspondence e-mail: \\ corrado.rizzoli@unipr.it
}

In a previous study, the $\mathrm{X}$-ray structure of the title compound, $\mathrm{C}_{9} \mathrm{H}_{12} \mathrm{~N}_{2} \mathrm{O}_{7} \mathrm{P}_{2} \cdot \mathrm{H}_{2} \mathrm{O}$, was reported [Takeuchi et al., (1998). Chem. Pharm. Bull. 46, 1703-1709], but neither atomic coordinates nor details of the geometry were published. The structure has been redetermined with high precision as its detailed knowledge is essential to elucidate the presumed polymorphism of minodronic acid monohydrate at room temperature. The molecule crystallizes in a zwitterionic form with cationic imidazolium[1,2a]pyridine and anionic phosphonate groups. The dihedral angle formed by the planes of the pyridine and imidazole rings is $3.55(9)^{\circ}$. A short intramolecular $\mathrm{C}-\mathrm{H} \cdots \mathrm{O}$ contact is present. In the crystal, molecules are linked by $\mathrm{O}-\mathrm{H} \cdots \mathrm{O}, \mathrm{N}-\mathrm{H} \cdots \mathrm{O}$ and $\mathrm{C}-$ $\mathrm{H} \cdot \mathrm{O} \mathrm{O}$ hydrogen bonds and $\pi-\pi$ interactions [centroid-to-centroid distance $=$ 3.5822 (11) $\AA$ ], forming a three-dimensional structure.

\section{Chemical context}

Minodronic acid, (1-hydroxy-2-(imidazo[1,2-a]pyridin-3yl)ethane-1,1-bis(phosphonic acid), has excellent bone resorption inhibitory activity, as well as anti-inflammatory, analgesic and antipyretic activities and is useful for the treatment of diseases in which an increased bone resorption participates (Tanishima \& Morio, 2013; Yamane et al., 2003; Ito et al., 2010; Sato et al., 2006). For practical uses and solid pharmaceutical preparations, the monohydrate form is preferred, giving more stable crystals with respect to the anhydrous and polyhydrate forms. By developing a pharmaceutical preparation (Takeuchi et al., 1993), it was found that monohydrate crystals include two crystal forms, named $D$ and $E$, having the same powder X-ray diffraction pattern but different dehydration temperatures. In fact, according to TGDSC thermogravimetric analyses, crystal $D$ has a dehydration peak temperature of 408 to $422 \mathrm{~K}$, whereas for $E$ it is 433 to $443 \mathrm{~K}$.

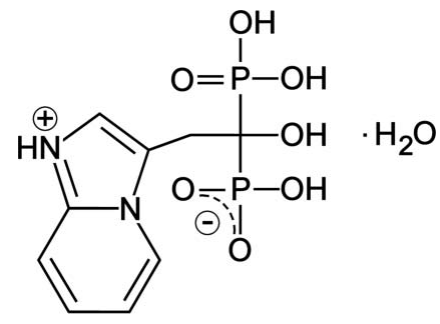




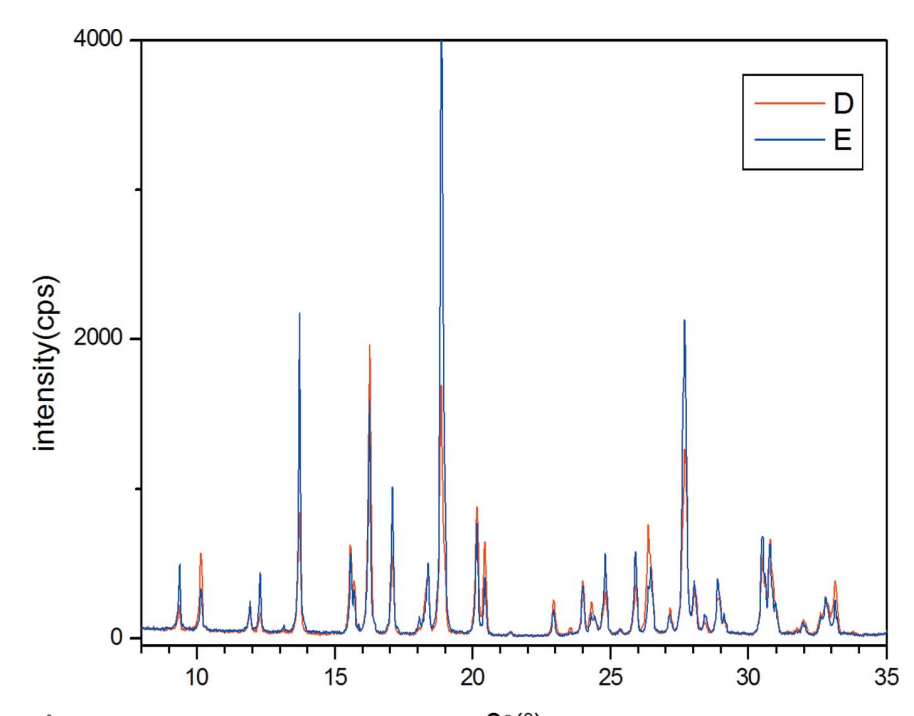

Figure 1 $2 \theta\left(^{\circ}\right)$

Comparison of the room-temperature powder X-ray diffraction patterns of forms $D$ (red line) and $E$ (blue line) of the title compound.

The monohydrate crystals of $D$ and $E$ are both produced by recrystallization of the free acid from aqueous hydrochloric acid solutions by gradual cooling of a heat-dissolved solution under mild stirring conditions, followed by drying the crystals at $313-333 \mathrm{~K}$ under reduced pressure. The stirring mode and cooling conditions are the key factors in determining the final crystal form.

The relation between the two crystal forms deviates from the conventional concept of polymorphism by the similarity of the powder X-ray diffraction patterns. In principle, different mechanisms could be invoked to justify the different thermal behavior, amongst which a small difference in the crystal packing or in the atomic interactions could lead to it. The crystal structure of a monohydrate form of minodronic acid has been published earlier (Takeuchi et al., 1998), but neither the atomic coordinates nor details of the molecular and crystal geometry were reported. In the absence of detailed information, the present study of the crystal structures of both forms, $D$ and $E$, was undertaken with the aim of finding a reasonable solution to the unusual 'polymorphism' problem. Batches of the $D$ and $E$ crystals were prepared as described in the Experimental section and characterized by thermogravimetric analysis.

Powder X-ray diffraction was performed with a Thermo X'tra Diffractometer equipped with a $\mathrm{Si}(\mathrm{Li})$ solid state detector directly on the as-prepared samples without grinding (Fig. 1). This confirmed that the diffraction patterns of the two forms involve peaks occurring at the same $2 \theta$ angles, the unique difference consisting of a different intensity distribution probably originating from preferential orientation. It is noteworthy that both diffraction patterns are fully compatible with the lattice parameters reported by Takeuchi et al. (1998). Small single crystals suitable for XRD experiments were selected from both batches and their structures solved and refined. The analyses established that the crystal structures are perfectly comparable within experimental error, and compatible with that previously reported.

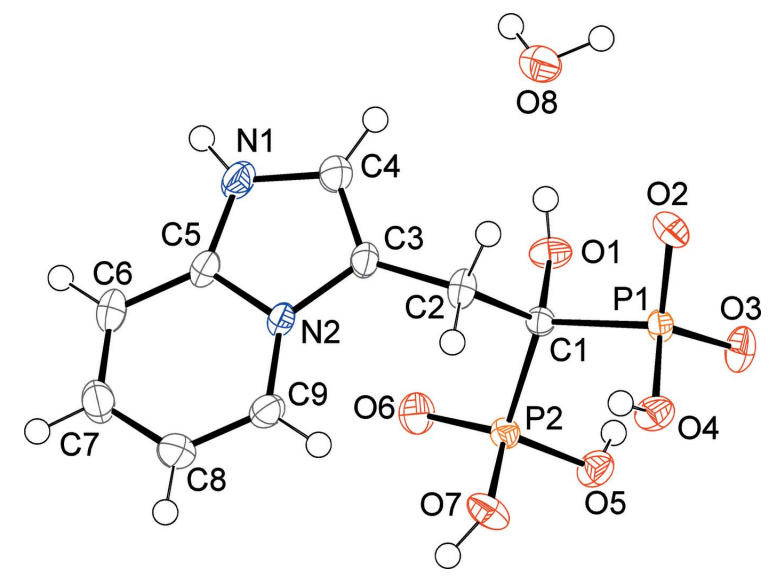

Figure 2

The molecular structure of the title compound, showing the atom labelling. Displacement ellipsoids are drawn at the $50 \%$ probability level.

We report herein the redetermination of the crystal structure of form $D$ of minodronic acid monohydrate, whereas that of form $E$ has been deposited at the CCDC (Rizzoli \& Calestani, 2014). On the basis of the present study, the peculiar thermal behaviour of the $D$ and $E$ forms of minodronic acid cannot be ascribed to structural differences, but it is probably due to morphological or microstructural features induced by the crystallization procedure and the influence of the dehydration process.

\section{Structural commentary}

The asymmetric unit of the title compound, Fig. 2, consists of minodronic acid and a water molecule of crystallization. The acid molecule crystallizes in a zwitterionic form with cationic imidazolium[1,2a]pyridine and anionic phosphonate groups. The fused-ring system deviates from planarity, with the dihedral angle formed by the pyridine and imidazole rings being $3.55(9)^{\circ}$. An analysis of the bond lengths within the fused-ring system, indicates that the $\mathrm{C}=\mathrm{N}$ and $\mathrm{C}=\mathrm{C}$ double-bond distribution shown in the Scheme is the most probable, but a resonant form involving the aromatic character of the pyridine ring and the localization of the positive change on the atom N2 also exists. The values of the $\mathrm{P}-\mathrm{O}$ bond lengths indicate that the negative charge is delocalized on atoms $\mathrm{O} 2$ and $\mathrm{O} 3$, whose distances $[\mathrm{P} 1-\mathrm{O} 2=1.5197(10), \mathrm{P} 1-\mathrm{O} 3=1.5001(10) \AA]$ are intermediate between those observed for the protonated atoms $\mathrm{O} 4, \mathrm{O} 5$ and $\mathrm{O} 7$ [mean value 1.564 (7) $\AA$ ] and for the localized $\mathrm{P}=\mathrm{O}$ double bond $[\mathrm{P} 2-\mathrm{O} 6=1.4817$ (10) $\AA$ ] . The observed distribution of the hydrogen atoms and of the charge on the phosphonate groups differs from that deducible from the molecular plot reported previously by Takeuchi et al. (1998). An intramolecular $\mathrm{C}-\mathrm{H} \cdots \mathrm{O}$ hydrogen bond is present (Table 1).

\section{Supramolecular features}

The crystal structure of the title compound is characterized by a very effective hydrogen-bonding network which is respon- 
Table 1

Hydrogen-bond geometry $\left(\AA,^{\circ}\right)$.

\begin{tabular}{|c|c|c|c|c|}
\hline$D-\mathrm{H} \cdots A$ & $D-\mathrm{H}$ & $\mathrm{H} \cdots A$ & $D \cdots A$ & $D-\mathrm{H} \cdots A$ \\
\hline $\mathrm{O} 1-\mathrm{H} 1 O \cdots \mathrm{O} 8$ & $0.78(3)$ & $1.87(3)$ & $2.6468(18)$ & $169(2)$ \\
\hline $\mathrm{O} 4-\mathrm{H} 4 O \cdots \mathrm{O} 2^{\mathrm{i}}$ & $0.90(3)$ & $1.73(3)$ & $2.6116(16)$ & $169(3)$ \\
\hline $\mathrm{O} 5-\mathrm{H} 5 O \cdots \mathrm{O}^{\mathrm{ii}}$ & $0.88(3)$ & $1.62(3)$ & $2.4973(14)$ & $175(3)$ \\
\hline $\mathrm{O} 7-\mathrm{H} 7 O \cdots \mathrm{O} 2^{\mathrm{iii}}$ & $0.83(2)$ & $1.80(2)$ & $2.6183(14)$ & $170(2)$ \\
\hline $\mathrm{O} 8-\mathrm{H} 81 \cdots \mathrm{O}^{\mathrm{iv}}$ & $0.82(2)$ & $1.99(2)$ & $2.8011(15)$ & $169(3)$ \\
\hline $\mathrm{O} 8-\mathrm{H} 82 \cdots \mathrm{O}^{\mathrm{v}}$ & $0.91(2)$ & $1.83(2)$ & $2.7334(16)$ & $175(2)$ \\
\hline $\mathrm{N} 1-\mathrm{H} 1 N \cdots \mathrm{O} 5^{\mathrm{vi}}$ & $0.85(2)$ & $2.18(3)$ & $2.9930(15)$ & $158(2)$ \\
\hline $\mathrm{C} 2-\mathrm{H} 2 B \cdots \mathrm{O} 2$ & 0.97 & 2.50 & $3.0224(17)$ & 114 \\
\hline $\mathrm{C} 4-\mathrm{H} 4 \ldots \mathrm{O} 8$ & 0.93 & 2.40 & 3.2079 (19) & 145 \\
\hline $\mathrm{C} 8-\mathrm{H} 8 \cdots \mathrm{O} 4^{\mathrm{vii}}$ & 0.93 & 2.51 & 3.1704 (17) & 128 \\
\hline
\end{tabular}

Symmetry codes: (i) $-x+1,-y,-z+1$; (ii) $-x+1,-y,-z$; (iii) $x-1, y, z$; (iv) $-x+1,-y+1,-z$; (v) $x+1, y, z$; (vi) $x, y+1, z$; (vii) $-x,-y,-z+1$.

sible for the unusually high value of the calculated density of the crystal $\left(1.818 \mathrm{~g} \mathrm{~cm}^{-3}\right)$. As shown in Fig. 3, the crystal packing may be described as an alternate stacking along the $b$ axis of phosphonate and organic layers forming a threedimensional network through $\mathrm{O}-\mathrm{H} \cdots \mathrm{O}, \mathrm{N}-\mathrm{H} \cdots \mathrm{O}$ and $\mathrm{C}-$ $\mathrm{H}$... O hydrogen bonds (Table 1 and Fig. 3). Adjacent phosphonate layers are bridged via hydrogen bonds involving the water molecules, which are hosted inside channels parallel to the $a$ axis and running between pairs of fused-ring systems connected by $\pi-\pi$ interactions $\left[C g 1 \cdots C g 1^{\mathrm{i}}=3.5822\right.$ (11) $\AA$; $C g 1$ is the centroid of the N2/C5-C9 ring; symmetry code: (i) $-x,-\mathrm{y}+1,-z+1]$. The $\mathrm{O}-\mathrm{H} \cdots \mathrm{O}$ hydrogen-bonding system within the phosphonate layer (Fig. 4) generates rings arranged in $R_{2}^{2}(8), R_{2}^{2}(12), R_{4}^{2}(16)$ and $R_{4}^{4}(16)$ graph-set motifs (Bernstein et al., 1995).

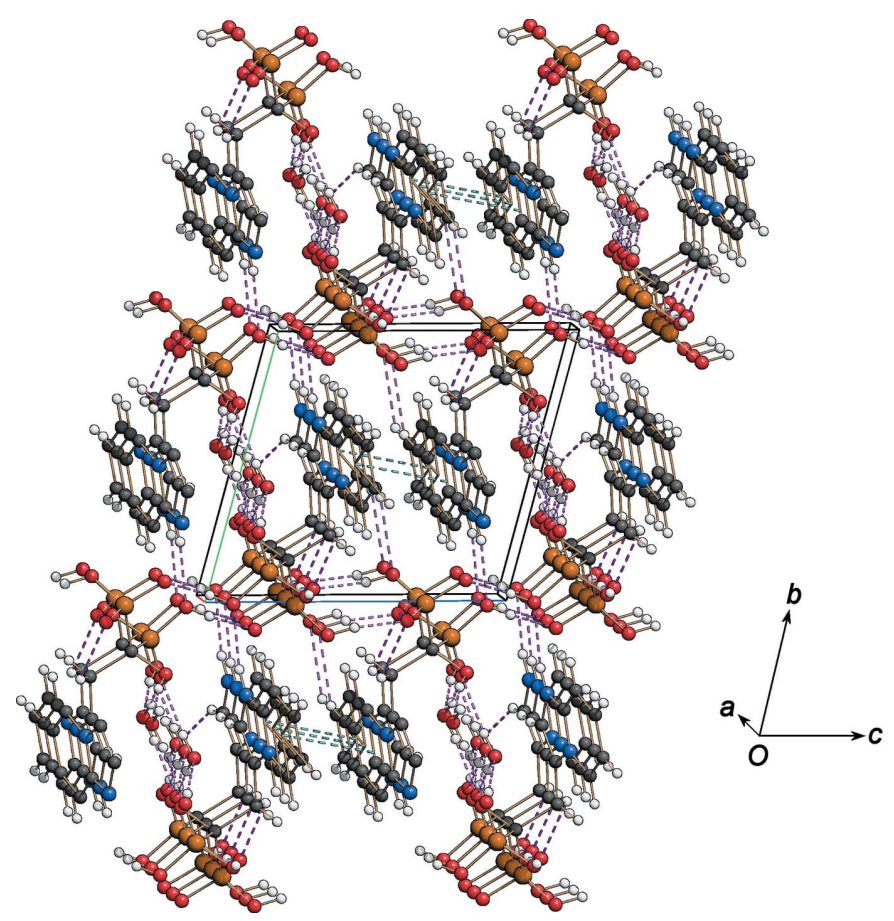

Figure 3

The crystal packing of the title compound viewed along the $a$ axis, showing the hydrogen-bonding network (violet dashed lines) and $\pi-\pi$ interactions (blue dashed lines); see Table 1 for details.

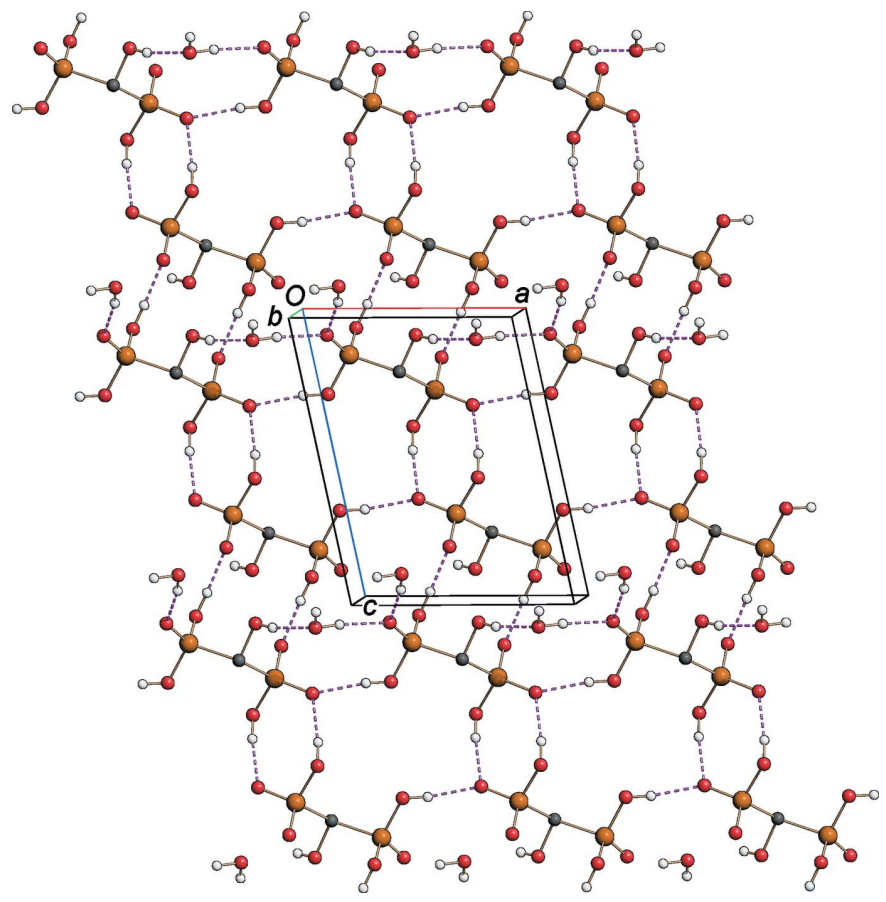

Figure 4

A view along the $b$ axis of the hydrogen-bonding network (dashed lines) in the phosphonate layer (see Table 1 for detail). Nitrogen, carbon (except $\mathrm{C} 1$ ) and $\mathrm{C}$ - and $\mathrm{N}$-bound $\mathrm{H}$ atoms have been omitted for clarity.

\section{Synthesis and crystallization}

Crystal form $D$ : crystals of minodronic acid (150 g) were added to 5.61 of $1 \mathrm{~N}$ hydrochloric acid (about 37.5 volumes) in a reactor equipped with mechanical stirring, and dissolved with heating. The dissolution temperature $(368 \mathrm{~K})$ was maintained for at least $30 \mathrm{~min}$, followed by filtration of a small amount of undissolved particles. The clear filtrate was stirred at 110 r.p.m. and slowly cooled to $328 \mathrm{~K}$ in $4 \mathrm{~h}$, and then to $295 \mathrm{~K}$ overnight. The crystalline precipitate was collected by filtration, washed with $300 \mathrm{ml}$ of water and $300 \mathrm{ml}$ of ethanol, and dried at $318 \mathrm{~K}$ to obtain $135 \mathrm{~g}$ of pure form $D$ of minodronic acid monohydrate, as shown by the dehydration peak at 415 K (Takeuchi et al., 1993). The colourless crystal sample used for X-ray analysis was selected from the batch.

Crystal form $E$ : crystals of minodronic acid (15 g) were added to 0.61 of $1 N$ hydrochloric acid (about 40 volumes) in a reactor equipped with magnetic stirring and dissolved with heating. The dissolution temperature $(368 \mathrm{~K})$ was maintained for at least $30 \mathrm{~min}$ followed by filtration of a small amount of undissolved particles. The clear filtrate was stirred slowly so that the liquid surface could not create a vortex (<110 r.p.m.) and gradually cooled down to $295 \mathrm{~K}$ overnight. The crystal precipitate was collected by filtration, washed with $30 \mathrm{ml}$ of water and $30 \mathrm{ml}$ of ethanol, and dried at $318 \mathrm{~K}$ to obtain $14 \mathrm{~g}$ of pure $E$ form of minodronic acid monohydrate, as shown by the dehydration peak at $438 \mathrm{~K}$ (Takeuchi et al., 1993). The colourless crystal sample used for X-ray analysis was selected from the batch. 
Table 2

Experimental details.

\begin{tabular}{|c|c|}
\hline \multicolumn{2}{|l|}{ Crystal data } \\
\hline Chemical formula & $\mathrm{C}_{9} \mathrm{H}_{12} \mathrm{~N}_{2} \mathrm{O}_{7} \mathrm{P}_{2} \cdot \mathrm{H}_{2} \mathrm{O}$ \\
\hline$M_{\mathrm{r}}$ & 340.16 \\
\hline Crystal system, space group & Triclinic, $P \overline{1}$ \\
\hline Temperature (K) & 294 \\
\hline$a, b, c(\AA)$ & $\begin{array}{l}7.3668(11), 8.9833(12) \\
9.9733(18)\end{array}$ \\
\hline$\alpha, \beta, \gamma\left({ }^{\circ}\right)$ & $\begin{array}{l}75.0136(17), 77.2716(17) \\
88.5706(18)\end{array}$ \\
\hline$V\left(\AA^{3}\right)$ & $621.54(17)$ \\
\hline$Z$ & 2 \\
\hline Radiation type & Мo $K \alpha$ \\
\hline$\mu\left(\mathrm{mm}^{-1}\right)$ & 0.40 \\
\hline Crystal size $(\mathrm{mm})$ & $0.18 \times 0.15 \times 0.10$ \\
\hline \multicolumn{2}{|l|}{ Data collection } \\
\hline Diffractometer & Bruker SMART 1000 CCD \\
\hline Absorption correction & $\begin{array}{l}\text { Multi-scan (SADABS; Bruker, } \\
\text { 2008) }\end{array}$ \\
\hline$T_{\min }, T_{\max }$ & $0.640,0.746$ \\
\hline $\begin{array}{l}\text { No. of measured, independent and } \\
\text { observed }[I>2 \sigma(I)] \text { reflections }\end{array}$ & $10459,4054,3413$ \\
\hline$R_{\text {int }}$ & 0.020 \\
\hline$(\sin \theta / \lambda)_{\max }\left(\AA^{-1}\right)$ & 0.752 \\
\hline \multicolumn{2}{|l|}{ Refinement } \\
\hline$R\left[F^{2}>2 \sigma\left(F^{2}\right)\right], w R\left(F^{2}\right), S$ & $0.035,0.101,1.07$ \\
\hline No. of reflections & 4054 \\
\hline No. of parameters & 218 \\
\hline $\mathrm{H}$-atom treatment & $\begin{array}{l}\mathrm{H} \text { atoms treated by a mixture of } \\
\text { independent and constrained } \\
\text { refinement }\end{array}$ \\
\hline$\Delta \rho_{\max }, \Delta \rho_{\min }\left(\mathrm{e} \AA^{-3}\right)$ & $0.55,-0.28$ \\
\hline
\end{tabular}

Computer programs: APEX2 and SAINT (Bruker, 2008), SIR97 (Altomare et al., 1999), SHELXL2014 (Sheldrick, 2008), ORTEP-3 for Windows (Farrugia, 2012), SCHAKAL99 (Keller, 1999) and publCIF (Westrip, 2010).

\section{Refinement}

Crystal data, data collection and structure refinement details are summarized in Table 2. All the $\mathrm{H}$ atoms were located in a difference Fourier map. The N- and O-bound hydrogen atoms were freely refined. The $\mathrm{C}$-bound $\mathrm{H}$ atoms were refined using a riding model approximation, with $\mathrm{C}-\mathrm{H}=0.93-0.97 \AA$ and with $U_{\text {iso }}(\mathrm{H})=1.2 U_{\text {eq }}(\mathrm{C})$.

\section{Acknowledgements}

The authors thank Mr A. Barozza, Dr J. Roletto and Dr P. Paissoni of PROCOS S.p.A. for useful discussions and valuable suggestions.

\section{References}

Altomare, A., Burla, M. C., Camalli, M., Cascarano, G. L., Giacovazzo, C., Guagliardi, A., Moliterni, A. G. G., Polidori, G. \& Spagna, R. (1999). J. Appl. Cryst. 32, 115-119.

Bernstein, J., Davis, R. E., Shimoni, L. \& Chang, N. L. (1995). Angew. Chem. Int. Ed. Engl. 34, 1555-1573.

Bruker (2008). APEX2, SAINT and SADABS. Bruker AXS Inc., Madison, Wisconsin, USA.

Farrugia, L. J. (2012). J. Appl. Cryst. 45, 849-854.

Ito, M., Sone, T. \& Fukunaga, M. (2010). J. Bone Miner. Metab. 28, 334-341.

Keller, E. (1999). SCHAKAL99. Universität Freiburg, Germany.

Rizzoli, C. \& Calestani, G. (2014). Private communication (deposition number 1037896). CCDC, Cambridge, England.

Sato, K., Yuasa, T., Nogawa, M., Kimura, S., Segawa, H., Yokota, A. \& Maekawa, T. (2006). Br. J. Cancer, 95, 1354-1361.

Sheldrick, G. M. (2008). Acta Cryst. A64, 112-122.

Takeuchi, M., Hamada, M., Kaneko, Y. \& Yamamoto, N. (1993). Eur. Patent EP 0647649B1.

Takeuchi, M., Sakamoto, S., Kawamuki, K., Kurihara, H., Nakahara, H. \& Isomura, Y. (1998). Chem. Pharm. Bull. 46, 1703-1709.

Tanishima, S. \& Morio, Y. (2013). Clin. Interv. Aging, 8, 185-189.

Westrip, S. P. (2010). J. Appl. Cryst. 43, 920-925.

Yamane, I., Hagino, H., Okano, T., Enokida, M., Yamasaki, D. \& Teshima, R. (2003). Arthritis Rheum. 48, 1732-1741. 


\section{supporting information}

Acta Cryst. (2015). E71, 51-54 [https://doi.org/10.1107/S2056989014026863]

Crystal structure of zwitterionic 3-(2-hydroxy-2-phosphonato-2-phosphonoethyl)imidazo[1,2-a]pyridin-1-ium monohydrate (minodronic acid monohydrate): a redetermination

Annalisa Airoldi, Piergiorgio Bettoni, Monica Donnola, Gianluca Calestani and Corrado Rizzoli

Computing details

Data collection: APEX2 (Bruker, 2008); cell refinement: SAINT (Bruker, 2008); data reduction: SAINT (Bruker, 2008); program(s) used to solve structure: SIR97 (Altomare et al., 1999); program(s) used to refine structure: SHELXL2014 (Sheldrick, 2008); molecular graphics: ORTEP-3 for Windows (Farrugia, 2012) and SCHAKAL99 (Keller, 1999); software used to prepare material for publication: publCIF (Westrip, 2010).

3-(2-Hydroxy-2-phosphonato-2-phosphonoethyl)imidazo[1,2-a]pyridin-1-ium monohydrate

Crystal data

$\mathrm{C}_{9} \mathrm{H}_{12} \mathrm{~N}_{2} \mathrm{O}_{7} \mathrm{P}_{2} \cdot \mathrm{H}_{2} \mathrm{O}$

$M_{r}=340.16$

Triclinic, $P \overline{1}$

$a=7.3668(11) \AA$

$b=8.9833(12) \AA$

$c=9.9733(18) \AA$

$\alpha=75.0136(17)^{\circ}$

$\beta=77.2716(17)^{\circ}$

$\gamma=88.5706(18)^{\circ}$

$V=621.54(17) \AA^{3}$

Data collection

Bruker SMART 1000 CCD diffractometer

$\omega$ scan

Absorption correction: multi-scan

(SADABS; Bruker, 2008)

$T_{\min }=0.640, T_{\max }=0.746$

10459 measured reflections

Refinement

Refinement on $F^{2}$

Least-squares matrix: full

$R\left[F^{2}>2 \sigma\left(F^{2}\right)\right]=0.035$

$w R\left(F^{2}\right)=0.101$

$S=1.07$

4054 reflections

218 parameters

0 restraints
$Z=2$

$F(000)=352$

$D_{\mathrm{x}}=1.818 \mathrm{Mg} \mathrm{m}^{-3}$

Mo $K \alpha$ radiation, $\lambda=0.71073 \AA$

Cell parameters from 227 reflections

$\theta=8.2-21.3^{\circ}$

$\mu=0.40 \mathrm{~mm}^{-1}$

$T=294 \mathrm{~K}$

Block, colourless

$0.18 \times 0.15 \times 0.10 \mathrm{~mm}$

4054 independent reflections

3413 reflections with $I>2 \sigma(I)$

$R_{\text {int }}=0.020$

$\theta_{\max }=32.3^{\circ}, \theta_{\min }=2.2^{\circ}$

$h=-11 \rightarrow 10$

$k=-12 \rightarrow 13$

$l=-14 \rightarrow 14$

Hydrogen site location: mixed

$\mathrm{H}$ atoms treated by a mixture of independent

and constrained refinement

$w=1 /\left[\sigma^{2}\left(F_{\mathrm{o}}^{2}\right)+(0.060 P)^{2}+0.1062 P\right]$

where $P=\left(F_{\mathrm{o}}{ }^{2}+2 F_{\mathrm{c}}{ }^{2}\right) / 3$

$(\Delta / \sigma)_{\max }=0.001$

$\Delta \rho_{\max }=0.55 \mathrm{e} \AA^{-3}$

$\Delta \rho_{\min }=-0.28$ e $\AA^{-3}$ 


\section{Special details}

Geometry. All e.s.d.'s (except the e.s.d. in the dihedral angle between two 1.s. planes) are estimated using the full covariance matrix. The cell e.s.d.'s are taken into account individually in the estimation of e.s.d.'s in distances, angles and torsion angles; correlations between e.s.d.'s in cell parameters are only used when they are defined by crystal symmetry. An approximate (isotropic) treatment of cell e.s.d.'s is used for estimating e.s.d.'s involving l.s. planes.

Fractional atomic coordinates and isotropic or equivalent isotropic displacement parameters $\left(\AA^{2}\right)$

\begin{tabular}{|c|c|c|c|c|}
\hline & $x$ & $y$ & $z$ & $U_{\text {iso }} * / U_{\text {eq }}$ \\
\hline P1 & $0.51328(4)$ & $-0.00236(4)$ & $0.28423(3)$ & $0.01430(9)$ \\
\hline $\mathrm{P} 2$ & $0.17313(4)$ & $0.13064(4)$ & $0.15681(3)$ & $0.01514(9)$ \\
\hline $\mathrm{O} 1$ & $0.49943(14)$ & $0.25308(12)$ & $0.08484(10)$ & $0.0206(2)$ \\
\hline $\mathrm{H} 1 \mathrm{O}$ & $0.576(3)$ & $0.303(3)$ & $0.098(2)$ & $0.039(6)^{*}$ \\
\hline $\mathrm{O} 2$ & $0.67125(13)$ & $0.05492(12)$ & $0.33555(10)$ & $0.0208(2)$ \\
\hline $\mathrm{O} 3$ & $0.56833(15)$ & $-0.09174(12)$ & $0.17446(11)$ & $0.0232(2)$ \\
\hline $\mathrm{O} 4$ & $0.37247(14)$ & $-0.10822(11)$ & $0.41416(11)$ & $0.0214(2)$ \\
\hline $\mathrm{H} 4 \mathrm{O}$ & $0.349(4)$ & $-0.080(3)$ & $0.496(3)$ & $0.070(8)^{*}$ \\
\hline O5 & $0.22204(14)$ & $0.01195(11)$ & $0.06740(11)$ & $0.0213(2)$ \\
\hline $\mathrm{H} 5 \mathrm{O}$ & $0.298(4)$ & $0.044(3)$ & $-0.016(3)$ & $0.072(8)^{*}$ \\
\hline O6 & $0.10307(14)$ & $0.27806(12)$ & $0.08260(12)$ & $0.0258(2)$ \\
\hline $\mathrm{O} 7$ & $0.03415(14)$ & $0.04399(13)$ & $0.29628(11)$ & $0.0240(2)$ \\
\hline $\mathrm{H} 7 \mathrm{O}$ & $-0.079(3)$ & $0.054(3)$ & $0.300(3)$ & $0.050(7)^{*}$ \\
\hline $\mathrm{O} 8$ & $0.77583(16)$ & $0.42957(14)$ & $0.09502(14)$ & $0.0301(3)$ \\
\hline H81 & $0.800(3)$ & $0.515(3)$ & $0.038(3)$ & $0.049(7)^{*}$ \\
\hline H82 & $0.884(3)$ & $0.379(3)$ & $0.086(2)$ & $0.049(6)^{*}$ \\
\hline N1 & $0.25482(18)$ & $0.67430(14)$ & $0.19284(13)$ & $0.0225(2)$ \\
\hline $\mathrm{H} 1 \mathrm{~N}$ & $0.277(3)$ & $0.769(3)$ & $0.148(2)$ & $0.047(6)^{*}$ \\
\hline N2 & $0.09082(15)$ & $0.46468(12)$ & $0.31543(12)$ & $0.0158(2)$ \\
\hline $\mathrm{C} 1$ & $0.38347(17)$ & $0.16943(14)$ & $0.21539(13)$ & $0.0138(2)$ \\
\hline $\mathrm{C} 2$ & $0.34325(19)$ & $0.26352(15)$ & $0.32898(14)$ & $0.0189(2)$ \\
\hline $\mathrm{H} 2 \mathrm{~A}$ & 0.2507 & 0.2061 & 0.4098 & $0.023^{*}$ \\
\hline $\mathrm{H} 2 \mathrm{~B}$ & 0.4565 & 0.2705 & 0.3619 & $0.023^{*}$ \\
\hline $\mathrm{C} 3$ & $0.27645(18)$ & $0.42267(15)$ & $0.28236(14)$ & $0.0176(2)$ \\
\hline $\mathrm{C} 4$ & $0.3748(2)$ & $0.55521(16)$ & $0.20766(15)$ & $0.0219(3)$ \\
\hline $\mathrm{H} 4$ & 0.5030 & 0.5637 & 0.1723 & $0.026^{*}$ \\
\hline $\mathrm{C} 5$ & $0.08150(19)$ & $0.62025(15)$ & $0.25700(14)$ & $0.0182(2)$ \\
\hline C6 & $-0.0888(2)$ & $0.69424(16)$ & $0.27288(16)$ & $0.0236(3)$ \\
\hline H6 & -0.0965 & 0.7984 & 0.2295 & $0.028^{*}$ \\
\hline $\mathrm{C} 7$ & $-0.2424(2)$ & $0.60818(17)$ & $0.35401(17)$ & $0.0260(3)$ \\
\hline $\mathrm{H} 7$ & -0.3572 & 0.6541 & 0.3670 & $0.031^{*}$ \\
\hline $\mathrm{C} 8$ & $-0.2293(2)$ & $0.44988(17)$ & $0.41874(16)$ & $0.0240(3)$ \\
\hline $\mathrm{H} 8$ & -0.3350 & 0.3937 & 0.4766 & $0.029^{*}$ \\
\hline C9 & $-0.06486(19)$ & $0.37863(15)$ & $0.39786(15)$ & $0.0200(3)$ \\
\hline H9 & -0.0576 & 0.2737 & 0.4385 & $0.024 *$ \\
\hline
\end{tabular}


Atomic displacement parameters $\left(\AA^{2}\right)$

\begin{tabular}{lllllll}
\hline & $U^{11}$ & $U^{22}$ & $U^{33}$ & $U^{12}$ & $U^{13}$ & $U^{23}$ \\
\hline P1 & $0.01367(15)$ & $0.01350(15)$ & $0.01500(15)$ & $0.00313(11)$ & $-0.00231(11)$ & $-0.00340(11)$ \\
P2 & $0.01371(15)$ & $0.01476(16)$ & $0.01648(16)$ & $0.00114(11)$ & $-0.00367(11)$ & $-0.00301(11)$ \\
O1 & $0.0207(5)$ & $0.0207(5)$ & $0.0165(4)$ & $-0.0069(4)$ & $-0.0002(4)$ & $-0.0004(4)$ \\
O2 & $0.0134(4)$ & $0.0274(5)$ & $0.0221(5)$ & $0.0026(4)$ & $-0.0045(4)$ & $-0.0072(4)$ \\
O3 & $0.0304(5)$ & $0.0192(5)$ & $0.0202(5)$ & $0.0075(4)$ & $-0.0029(4)$ & $-0.0084(4)$ \\
O4 & $0.0239(5)$ & $0.0183(5)$ & $0.0185(4)$ & $-0.0037(4)$ & $-0.0016(4)$ & $-0.0008(4)$ \\
O5 & $0.0248(5)$ & $0.0190(5)$ & $0.0213(5)$ & $-0.0009(4)$ & $-0.0038(4)$ & $-0.0084(4)$ \\
O6 & $0.0243(5)$ & $0.0199(5)$ & $0.0327(6)$ & $0.0059(4)$ & $-0.0123(4)$ & $-0.0016(4)$ \\
O7 & $0.0138(5)$ & $0.0324(6)$ & $0.0216(5)$ & $-0.0010(4)$ & $-0.0005(4)$ & $-0.0024(4)$ \\
O8 & $0.0204(5)$ & $0.0258(6)$ & $0.0404(7)$ & $-0.0019(4)$ & $-0.0044(5)$ & $-0.0036(5)$ \\
N1 & $0.0259(6)$ & $0.0131(5)$ & $0.0244(6)$ & $-0.0003(4)$ & $0.0008(5)$ & $-0.0028(4)$ \\
N2 & $0.0186(5)$ & $0.0112(5)$ & $0.0179(5)$ & $0.0024(4)$ & $-0.0037(4)$ & $-0.0044(4)$ \\
C1 & $0.0132(5)$ & $0.0131(5)$ & $0.0142(5)$ & $0.0004(4)$ & $-0.0017(4)$ & $-0.0028(4)$ \\
C2 & $0.0235(6)$ & $0.0156(6)$ & $0.0192(6)$ & $0.0063(5)$ & $-0.0070(5)$ & $-0.0060(5)$ \\
C3 & $0.0188(6)$ & $0.0149(6)$ & $0.0198(6)$ & $0.0035(4)$ & $-0.0034(5)$ & $-0.0070(5)$ \\
C4 & $0.0206(6)$ & $0.0193(6)$ & $0.0247(7)$ & $0.0010(5)$ & $-0.0007(5)$ & $-0.0077(5)$ \\
C5 & $0.0236(6)$ & $0.0121(5)$ & $0.0183(6)$ & $0.0021(4)$ & $-0.0033(5)$ & $-0.0040(4)$ \\
C6 & $0.0277(7)$ & $0.0164(6)$ & $0.0273(7)$ & $0.0077(5)$ & $-0.0080(6)$ & $-0.0059(5)$ \\
C7 & $0.0221(7)$ & $0.0264(7)$ & $0.0324(7)$ & $0.0079(5)$ & $-0.0074(6)$ & $-0.0121(6)$ \\
C8 & $0.0197(6)$ & $0.0240(7)$ & $0.0271(7)$ & $-0.0017(5)$ & $-0.0015(5)$ & $-0.0075(5)$ \\
C9 & $0.0222(6)$ & $0.0153(6)$ & $0.0211(6)$ & $-0.0018(5)$ & $-0.0029(5)$ & $-0.0038(5)$ \\
& & & & & & \\
\hline & & & & & &
\end{tabular}

Geometric parameters $\left(\AA,{ }^{\circ}\right)$

\begin{tabular}{llll}
\hline $\mathrm{P} 1-\mathrm{O} 3$ & $1.5001(10)$ & $\mathrm{N} 2-\mathrm{C} 9$ & $1.3743(17)$ \\
$\mathrm{P} 1-\mathrm{O} 2$ & $1.5197(10)$ & $\mathrm{N} 2-\mathrm{C} 5$ & $1.3744(16)$ \\
$\mathrm{P} 1-\mathrm{O} 4$ & $1.5745(10)$ & $\mathrm{N} 2-\mathrm{C} 3$ & $1.4004(17)$ \\
$\mathrm{P} 1-\mathrm{C} 1$ & $1.8534(13)$ & $\mathrm{C} 1-\mathrm{C} 2$ & $1.5558(17)$ \\
$\mathrm{P} 2-\mathrm{O} 6$ & $1.4817(10)$ & $\mathrm{C} 2-\mathrm{C} 3$ & $1.4914(18)$ \\
$\mathrm{P} 2-\mathrm{O} 5$ & $1.5468(10)$ & $\mathrm{C} 2-\mathrm{H} 2 \mathrm{~A}$ & 0.9700 \\
$\mathrm{P} 2-\mathrm{O} 7$ & $1.5625(10)$ & $\mathrm{C} 2-\mathrm{H} 2 \mathrm{~B}$ & 0.9700 \\
$\mathrm{P} 2-\mathrm{C} 1$ & $1.8426(13)$ & $\mathrm{C} 3-\mathrm{C} 4$ & $1.3580(19)$ \\
$\mathrm{O} 1-\mathrm{C} 1$ & $1.4233(15)$ & $\mathrm{C} 4-\mathrm{H} 4$ & 0.9300 \\
$\mathrm{O} 1-\mathrm{H} 1 \mathrm{O}$ & $0.78(2)$ & $\mathrm{C} 5-\mathrm{C} 6$ & $1.4022(19)$ \\
$\mathrm{O} 4-\mathrm{H} 4 \mathrm{O}$ & $0.90(3)$ & $\mathrm{C} 6-\mathrm{C} 7$ & $1.360(2)$ \\
$\mathrm{O} 5-\mathrm{H} 5 \mathrm{O}$ & $0.88(3)$ & $\mathrm{C} 6-\mathrm{H} 6$ & 0.9300 \\
$\mathrm{O} 7-\mathrm{H} 7 \mathrm{O}$ & $0.83(2)$ & $\mathrm{C} 7-\mathrm{C} 8$ & $1.412(2)$ \\
$\mathrm{O} 8-\mathrm{H} 81$ & $0.83(3)$ & $\mathrm{C} 7-\mathrm{H} 7$ & $1.3560(2)$ \\
$\mathrm{O} 8-\mathrm{H} 82$ & $0.91(2)$ & $\mathrm{C} 8-\mathrm{C} 9$ & 0.9300 \\
$\mathrm{~N} 1-\mathrm{C} 5$ & $\mathrm{C} 9-\mathrm{H} 9$ & 0.9300 \\
$\mathrm{~N} 1-\mathrm{C} 4$ & $1.3363(18)$ & & $115.68(7)$ \\
$\mathrm{N} 1-\mathrm{H} 1 \mathrm{~N}$ & $1.3700(18)$ & $\mathrm{P} 2-\mathrm{C} 1-\mathrm{P} 1$ & $116.39(11)$ \\
$\mathrm{O} 3-\mathrm{P} 1-\mathrm{O} 2$ & $0.85(2)$ & $\mathrm{C} 3-\mathrm{C} 2-\mathrm{C} 1$ &
\end{tabular}




$\begin{array}{llll}\mathrm{O} 2-\mathrm{P} 1-\mathrm{O} 4 & 109.85(6) & \mathrm{C} 3-\mathrm{C} 2-\mathrm{H} 2 \mathrm{~A} & 108.2 \\ \mathrm{O} 3-\mathrm{P} 1-\mathrm{C} 1 & 110.32(6) & \mathrm{C} 1-\mathrm{C} 2-\mathrm{H} 2 \mathrm{~A} & 108.2 \\ \mathrm{O} 2-\mathrm{P} 1-\mathrm{C} 1 & 106.68(6) & \mathrm{C} 3-\mathrm{C} 2-\mathrm{H} 2 \mathrm{~B} & 108.2 \\ \mathrm{O} 4-\mathrm{P} 1-\mathrm{C} 1 & 106.17(6) & \mathrm{C} 1-\mathrm{C} 2-\mathrm{H} 2 \mathrm{~B} & 108.2 \\ \mathrm{O} 6-\mathrm{P} 2-\mathrm{O} 5 & 115.07(6) & \mathrm{H} 2 \mathrm{~A}-\mathrm{C} 2-\mathrm{H} 2 \mathrm{~B} & 107.3 \\ \mathrm{O} 6-\mathrm{P} 2-\mathrm{O} 7 & 113.46(6) & \mathrm{C} 4-\mathrm{C}-\mathrm{N} 2 & 129.72(11) \\ \mathrm{O} 5-\mathrm{P} 2-\mathrm{O} 7 & 105.64(6) & \mathrm{C} 4-\mathrm{C} 3-\mathrm{C} 2 & 124.62(12) \\ \mathrm{O} 6-\mathrm{P} 2-\mathrm{C} 1 & 109.47(6) & \mathrm{N} 2-\mathrm{C} 3-\mathrm{C} 2 & 109.01(13) \\ \mathrm{O} 5-\mathrm{P} 2-\mathrm{C} 1 & 107.81(6) & \mathrm{C} 3-\mathrm{C} 4-\mathrm{N} 1 & 125.5 \\ \mathrm{O} 7-\mathrm{P} 2-\mathrm{C} 1 & 104.75(6) & \mathrm{C} 3-\mathrm{C} 4-\mathrm{H} 4 & 125.5 \\ \mathrm{C} 1-\mathrm{O} 1-\mathrm{H} 1 \mathrm{O} & 111.6(16) & \mathrm{N} 1-\mathrm{C} 4-\mathrm{H} 4 & 107.39(11) \\ \mathrm{P} 1-\mathrm{O} 4-\mathrm{H} 4 \mathrm{O} & 116.9(17) & \mathrm{N} 1-\mathrm{C} 5-\mathrm{N} 2 & 131.56(13) \\ \mathrm{P} 2-\mathrm{O} 5-\mathrm{H} 5 \mathrm{O} & 116.7(18) & \mathrm{N} 1-\mathrm{C} 5-\mathrm{C} 6 & 11.04(12) \\ \mathrm{P} 2-\mathrm{O} 7-\mathrm{H} 7 \mathrm{O} & 118.0(17) & \mathrm{N} 2-\mathrm{C} 5-\mathrm{C} 6 & 121.2 \\ \mathrm{H} 81-\mathrm{O} 8-\mathrm{H} 82 & 105(2) & \mathrm{C} 7-\mathrm{C} 6-\mathrm{C} 5 & 121.2 \\ \mathrm{C} 5-\mathrm{N} 1-\mathrm{C} 4 & 109.26(12) & \mathrm{C} 7-\mathrm{C} 6-\mathrm{H} 6 & 120.72(13) \\ \mathrm{C} 5-\mathrm{N} 1-\mathrm{H} 1 \mathrm{~N} & 121.0(15) & \mathrm{C} 5-\mathrm{C} 6-\mathrm{H} 6 & 119.6 \\ \mathrm{C} 4-\mathrm{N} 1-\mathrm{H} 1 \mathrm{~N} & 129.6(15) & \mathrm{C} 6-\mathrm{C} 7-\mathrm{C} 8 & 119.6 \\ \mathrm{C} 9-\mathrm{N} 2-\mathrm{C} 5 & 120.93(11) & \mathrm{C} 6-\mathrm{C} 7-\mathrm{H} 7 & 120.97(13) \\ \mathrm{C} 9-\mathrm{N} 2-\mathrm{C} 3 & 130.37(11) & \mathrm{C} 8-\mathrm{C} 7-\mathrm{H} 7 & 119.5 \\ \mathrm{C} 5-\mathrm{N} 2-\mathrm{C} 3 & 108.59(11) & \mathrm{C} 9-\mathrm{C} 8-\mathrm{C} 7 & 119.5 \\ \mathrm{O} 1-\mathrm{C} 1-\mathrm{C} 2 & 112.48(10) & \mathrm{C} 9-\mathrm{C} 8-\mathrm{H} 8 & 118.63(12) \\ \mathrm{O} 1-\mathrm{C} 1-\mathrm{P} 2 & 101.50(8) & \mathrm{C} 7-\mathrm{C} 8-\mathrm{H} 8 & 120.7 \\ \mathrm{C} 2-\mathrm{C} 1-\mathrm{P} 2 & 112.69(8) & \mathrm{C} 8-\mathrm{C} 9-\mathrm{N} 2 & \\ \mathrm{O} 1-\mathrm{C} 1-\mathrm{P} 1 & 106.38(8) & \mathrm{C} 8-\mathrm{C} 9-\mathrm{H} 9 & \mathrm{~N} 9 \\ \mathrm{C} 2-\mathrm{C} 1-\mathrm{P} 1 & 107.91(8) & \mathrm{N} 2-\mathrm{C} 9-\mathrm{H} 9 & \\ & & & \end{array}$

Hydrogen-bond geometry $\left(A,{ }^{\circ}\right)$

\begin{tabular}{|c|c|c|c|c|}
\hline$D-\mathrm{H} \cdots A$ & $D-\mathrm{H}$ & $\mathrm{H} \cdots A$ & $D \cdots A$ & $D-\mathrm{H} \cdots A$ \\
\hline $\mathrm{O} 1-\mathrm{H} 1 O \cdots \mathrm{O} 8$ & $0.78(3)$ & $1.87(3)$ & $2.6468(18)$ & $169(2)$ \\
\hline $\mathrm{O} 4-\mathrm{H} 4 O \cdots \mathrm{O} 2^{\mathrm{i}}$ & $0.90(3)$ & $1.73(3)$ & $2.6116(16)$ & $169(3)$ \\
\hline $\mathrm{O} 5-\mathrm{H} 5 O \cdots \mathrm{O}^{\mathrm{ii}}$ & $0.88(3)$ & $1.62(3)$ & $2.4973(14)$ & $175(3)$ \\
\hline $\mathrm{O} 7-\mathrm{H} 7 O^{\cdots} \mathrm{O} 2^{\mathrm{iii}}$ & $0.83(2)$ & $1.80(2)$ & $2.6183(14)$ & $170(2)$ \\
\hline $\mathrm{O} 8-\mathrm{H} 81 \cdots \mathrm{O} 6^{\mathrm{iv}}$ & $0.82(2)$ & $1.99(2)$ & $2.8011(15)$ & $169(3)$ \\
\hline $\mathrm{O} 8-\mathrm{H} 82 \cdots \mathrm{O}^{\mathrm{v}}$ & $0.91(2)$ & $1.83(2)$ & $2.7334(16)$ & $175(2)$ \\
\hline $\mathrm{N} 1-\mathrm{H} 1 N \cdots \mathrm{O} 5^{\mathrm{vi}}$ & $0.85(2)$ & $2.18(3)$ & $2.9930(15)$ & $158(2)$ \\
\hline $\mathrm{C} 2-\mathrm{H} 2 B \cdots \mathrm{O} 2$ & 0.97 & 2.50 & $3.0224(17)$ & 114 \\
\hline $\mathrm{C} 4-\mathrm{H} 4 \cdots \mathrm{O} 8$ & 0.93 & 2.40 & $3.2079(19)$ & 145 \\
\hline $\mathrm{C} 8-\mathrm{H} 8 \cdots \mathrm{O} 4^{\mathrm{vii}}$ & 0.93 & 2.51 & $3.1704(17)$ & 128 \\
\hline
\end{tabular}

Symmetry codes: (i) $-x+1,-y,-z+1$; (ii) $-x+1,-y,-z$; (iii) $x-1, y, z$; (iv) $-x+1,-y+1,-z$; (v) $x+1, y, z$; (vi) $x, y+1, z$; (vii) $-x,-y,-z+1$. 